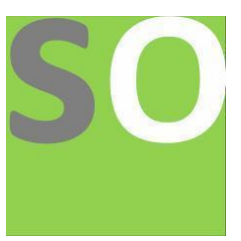

Article title: Twin Prime Conjecture - Proof that there are infinite twin primes.

Authors: Vijay Nandanwar[1]

Affiliations: Independent, Non-affiliated[1]

Orcid ids: 0000-0002-6313-1943[1]

Contact e-mail: vijaynandanwar2003@gmail.com

License information: This work has been published open access under Creative Commons Attribution License http://creativecommons.org/licenses/by/4.0/, which permits unrestricted use, distribution, and reproduction in any medium, provided the original work is properly cited. Conditions, terms of use and publishing policy can be found at https://www.scienceopen.com/.

Preprint statement: This article is a preprint and has not been peer-reviewed, under consideration and submitted to ScienceOpen Preprints for open peer review.

DOI: 10.14293/S2199-1006.1.SOR-.PPKAVE2.v1

Preprint first posted online: 20 August 2021

Keywords: Twin Prime Conjecture, Number Theory, Infinite Twin Primes 


\title{
TWIN PRIME CONJECTURE - PROOF THAT THERE ARE INFINITE TWIN PRIMES
}

\author{
VIJAY NANDANWAR
}

August 19, 2021

\begin{abstract}
We determine that twin primes are not random within specific range of number series. Within the range, they are part of an infinite repeating cycle within the series. The repeating cycles of this series is symmetrical and that range is part of one such cycle. We can also determine where in the repeating cycle this range is located. Once we establish the repeating cycles, we find the probability of twin primes for the cycle/series. Next we determine the lower bound for the probability and the range. Finally, we prove that the twin prime conjecture is true.
\end{abstract}

\section{INTRODUCTION}

Twin prime numbers are prime number pairs that are separated by 2 ; e.g. $(3,5)$, $(5,7),(11,13),(17,19),(29,31)$ and so on. Twin prime conjecture states that there are infinite number of such pairs. Though this is generally accepted to be true, and lots of progress has been made in recent years towards proving this conjecture, at present there is no proof for the same.

Since we are discussing twin primes, we will consider odd numbers $>=3$. We will go in a step by step process towards the proof and build up the justifications along the way.

\section{TWIN PRIMES WITHIN A RANGE}

\section{Get number of potential twin primes}

Without applying the impact of any odd prime number, for any odd number $N>3$, we have the maximum potential $\frac{N+1}{2}-3$ twin primes upto $\mathrm{N}$, but not including N. For example, 
Maximum potential twin primes Examples

$$
\begin{array}{rrrc}
N=5 & \frac{5+1}{2}-3 & 0 & \\
N=7 & \frac{7+1}{2}-3 & 1 & (3,5) \\
N=9 & \frac{9+1}{2}-3 & 2 & (3,5),(5,7) \\
\text { and so on } \ldots & & &
\end{array}
$$

The above treats all odd numbers as prime numbers. So, the maximum number of potential twin primes for an odd number $N>3$ upto $\mathrm{N}$, but not including $\mathrm{N}$ can be defined as

$$
\operatorname{PTP}(N)=\frac{N+1}{2}-3
$$

\section{Calculate the impact of introducing a prime number on potential twin primes}

Here we will determine the impact of introducing a prime number on potential twin primes series. We need to keep the following in mind:

- Non-prime odd numbers do not have any impact on the series. The prime factors of the non-primes have already taken care of the multiples in the series

\begin{tabular}{|c|c|c|}
\hline Prime Number & Impact Starts at & Explanation \\
\hline 3 & 9 & Impact of 3 first starts at 9 \\
\hline 5 & 25 & $\begin{array}{l}\text { Impact of } 5 \text { first starts at } 25 \text {, before } \\
\text { that it is taken care of by } 3 \text {; e.g.; } 15 \\
\text { is already accounted for by } 3\end{array}$ \\
\hline 7 & 49 & $\begin{array}{l}\text { Impact of } 7 \text { first starts at } 49 \text {, before that } \\
\text { it is taken care of by } 3(21) \text { or } 5(35)\end{array}$ \\
\hline 9 & No Impact & $\begin{array}{l}\text { No Impact of } 9 \text {, all multiples already } \\
\text { taken care of by } 3\end{array}$ \\
\hline
\end{tabular}

- The impact of introducing a prime number $\mathrm{p}$ to the series only starts from $p^{2}$ onwards as cases before that are already taken care of by one of the earlier smaller prime number

For example:

and so on ...

It is quite possible that first impact on potential twin primes series may happen after $p^{2}$ in case both $p^{2}-2$ and $p^{2}+2$ have already been taken care of by earlier primes. For example, $11^{2}=121$ and $119=7^{*} 17$ and $123=3^{*} 41$, but it does not matter. For our calculations, we still take $p^{2}$ as the effective starting value, since it would have been prime otherwise. 


\section{Impact of introducing 3 on potential twin primes}

$\begin{array}{cl}\text { Twin Prime } & \text { Impact } \\ (7,9) & \text { Yes } \\ (9,11) & \text { Yes } \\ (11,13) & \text { No Impact } \\ (13,15) & \text { Yes } \\ (15,17) & \text { Yes } \\ (17,19) & \text { No Impact } \\ (19,21) & \text { Yes } \\ (21,23) & \text { Yes } \\ \text { and so on } \ldots & \end{array}$

By introducing multiples of 3 as composite, it has affected potential twin primes on either side of the composite number. So we define I(3) as "Impact of 3 on the remaining potential twin primes $>=9$ " in the number series as

$$
I(3)=\frac{2}{3}
$$

$\mathrm{P}(3)$ defined as "Probability of finding a potential twin prime after impact of 3 (which first starts at 9 as stated earlier)

$$
P(3)=1-I(3)=1-\frac{2}{3}=\frac{1}{3}
$$

- Without the impact of 3 , i.e. for numbers less than 9 , the probability of finding twin primes is 1 . These are $(3,5)$ and $(5,7)$

- After the impact of 3 is considered, there are no more overlapping twin primes left

- Introduction of any new prime number will only impact one potential twin prime (by impacting one number of the potential twin prime) at a time from here onwards

\section{Impact of introducing 5 on remaining potential twin primes}

We need to get the whole cycle to assess the impact of introducing 5 . To get one full cycle, we consider the numbers from $3^{*} 5^{*} 1$ to $3^{*} 5^{*} 3$ (a range of $3^{*} 5^{*} 2$ ), i.e., from 15 to 45

We notice that there are 5 potential twin primes left after introducing 3 . Once we introduce 5 :

$\begin{array}{cl}\text { Twin Prime } & \text { Impact } \\ (17,19) & \text { No Impact } \\ (23,25) & \text { Yes } \\ (29,31) & \text { No Impact } \\ (35,37) & \text { Yes } \\ (41,43) & \text { No Impact }\end{array}$


By introducing multiples of 5 as composite, it has affected remaining potential twin primes when any one of the numbers of the potential twin prime is impacted. So I(5) can be calculated as

$$
I(5)=\frac{2}{5} * P(3)
$$

Similarty $\mathrm{P}(5)$ can be calculated as

$$
P(5)=P(3)-I(5)=P(3)-\frac{2}{5} * P(3)
$$

\section{Impact of introducing 7 on remaining potential twin primes}

Here, to get the full cycle, we need to consider the numbers from $3 * 5^{*} 7^{*} 1$ to $3^{*} 5^{*} 7^{*} 3$ (as range of $3 * 5^{*} 7^{*} 2$ ), i.e. from 105 to 315 . Using the same process as above, we can derive the following

$$
\begin{gathered}
I(7)=\frac{2}{7} * P(5) \\
P(7)=P(5)-I(7)=P(5)-\frac{2}{7} * P(5)
\end{gathered}
$$

So we notice a pattern emerging here as shown below ( $T P=$ TwinPrime $)$ :

$$
\text { Prime One Cycle TPs TPs Proportion }
$$

$\begin{array}{ccccc}\text { Introduction } & & \text { Before } & \text { After } & \text { Impacted } \\ 3 & 9-15 & 3 & 1 & \frac{2}{3} \\ 5 & 15-45 & 5 & 3 & \frac{2}{5} \\ 7 & 105-315 & 21 & 15 & \frac{2}{7} \\ 11 & 1155-3465 & 165 & 135 & \frac{2}{11} \\ 13 & 15015-45045 & 1755 & 1485 & \frac{2}{13}\end{array}$

and so on ...

For 3 , we take the range from $9-15\left(3^{*} 3-3^{*} 5\right)$, because the first time any prime number occurs, it is treated as part of a twin prime even though it is completely divisible by itself. We can take the range from any other consecutive odd multiple later in the series too, not just for 3 but also for other prime numbers. 


\section{What repeats in the series from a cycle}

The remainder of the number when divided by the introduced prime number in a cycle repeats in the series.

$\begin{array}{ccccccccccccccc}\text { Prime } & 1 & 3 & 5 & 7 & 9 & 11 & 13 & 15 & 17 & 19 & 21 & 23 & 25 & 27 \\ 3 & 1 & 0 & 2 & 1 & 0 & 2 & 1 & 0 & 2 & 1 & 0 & 2 & 1 & 0 \\ 5 & 1 & 3 & 0 & 2 & 4 & 1 & 3 & 0 & 2 & 4 & 1 & 3 & 0 & 2\end{array}$

and so on ...

\section{Symmetry within each cycle before the introduction of higher prime numbers}

The reason we choose the range for a cycle within the series as we do is because we are looking at a place to start afresh. And that would be the Lowest Common Multiple (LCM) of all the odd primes that came before it. The remainder is 0 for that number for all the prime numbers introduced. The next time the remainder is 0 is when we multiply the LCM by 3 . This determines our range for the cycle, which gets repeated again and again. Also a quick observation shows that within the range there is symmetry of the potential twin primes around the center (multiplication by 2). This means that within each cycle, the distribution of remainders being 0 or not 0 for the prime numbers (individually or taken together) is symmetrical.

Prime number cumulative multiplication is a faster growing function than the square of prime numbers

By this we mean that $\prod_{p} p_{i}$ is a faster growing function than $p_{i+1}^{2}$. This means that the actual range that we will use is well contained within a cycle. However, there is an intersection. For smaller numbers, cumulative multiplication is less, but it soon catches up and then grows faster forever. This is covered more later.

General Impact of introducing any prime number on the remaining potential twin primes

Normally, one would expect that introducing a prime number, say $\mathrm{p}$, would impact one out of every p remaining number. However, twin primes are two different primes together, so any one (and only one) of these can be impacted, either the left one or the right one, and that would mean that that potential twin prime is impacted. So 2 in the numerator is expected. This can also be expected because of the symmetry. If one of the potential twin primes is impacted, then the corresponding symmetrical potential twim prime would also be impacted in the cycle.

We are finally ready to write general probability equations. Let us denote a set of $n$ prime numbers by

$$
p_{1}, p_{2}, \ldots, p_{n}
$$


We write our general equation as

$$
P\left(p_{i}\right)=P\left(p_{i-1}\right)-\frac{2}{p_{i}} * P\left(p_{i-1}\right)
$$

or

$$
P\left(p_{i}\right)=\frac{\left(p_{i}-2\right)}{p_{i}} * P\left(p_{i-1}\right)
$$

With this equation, we can calculate the probability for the next potential twin prime number based on the probability of the previous potential twin prime number. We can write it as

$$
P\left(p_{i}\right)=\frac{\left(p_{i}-2\right)}{p_{i}} * \frac{\left(p_{i-1}-2\right)}{p_{i-1}} * \ldots * \frac{(11-2)}{11} * \frac{(7-2)}{7} * \frac{(5-2)}{5} * \frac{(3-2)}{3}
$$

It is worth noting that we skip 9 (we skip all composite odd numbers). And the effective range for this probability is from

$$
>=p_{i}^{2} \text { and }<p_{i+1}^{2}
$$

Prime Number

3

5

7

11

and so on ...
Probability

$$
\begin{array}{cc}
\frac{3-2}{3}=\frac{1}{3} ; \text { from earlier } & >=9 \text { and }<25 \\
\frac{5-2}{5} * \frac{3-2}{3}=\frac{1}{5} & >=25 \text { and }<49 \\
\frac{7-2}{7} * \frac{5-2}{5} * \frac{3-2}{3}=\frac{1}{7} & >=49 \text { and }<121 \\
\frac{11-2}{11} * \frac{7-2}{7} * \frac{5-2}{5} * \frac{3-2}{3}=\frac{1}{11} * \frac{9}{7} & >=121 \text { and }<169
\end{array}
$$

Effective Range

\section{Twin prime vs Potential twin prime}

So far we have been talking about potential twin primes, meaning these can be impacted. Within any effective range, the potential twin primes that are not impacted are the twin primes. The ones that are impacted no longer remain twin primes.

\section{Where in the cycle does our effective range lie}

Our effective range lies to the right of the center of the cycle. Meaning one such range can fall from $\left(\prod_{p} p_{i} * 2+\right.$ Lower bound of effective range) (inclusive) to $\left(\prod_{p} p_{i} * 2+\right.$ Upper bound of effective range) (exclusive).

\section{GETTING THE EXPECTED NUMBER OF TWIN PRIMES}

Now that we have the probabilities and the ranges, we can multiply these two and calculate the expected number of twin primes for any of these ranges. 


$\begin{array}{ccccc}\text { Prime Number } & \text { Probability } & \text { Effective Range } & \text { PTPs } & \text { Estimated TPs } \\ 3 & \frac{1}{3} \text {; from earlier } & >=9 \text { and }<25 & 7 & 2.33 \\ 5 & \frac{1}{5} & >=25 \text { and }<49 & 11 & 2.2 \\ 7 & \frac{1}{7} & >=49 \text { and }<121 & 35 & 5 \\ 11 & \frac{1}{11} * \frac{9}{7} & >=121 \text { and }<169 & 23 & 2.69 \\ 13 & \frac{1}{13} * \frac{9}{7} & >=169 \text { and }<289 & 59 & 5.84 \\ \text { and so on ... } & & & & \end{array}$

The above does not give us the exact number, sometimes more and sometimes less. This is because within a cycle, even though the potential twin primes are symmetrically distributed, they are not evenly distributed. The actual number depends on the part of the cycle that the range belongs to. Also as the number increases and the distance between consecutive prime numbers, the estimated number of twin primes also increase. However, here we are interested in proving that there are infinite number of twin primes, which brings us to the next section.

\section{GETTING THE LOWER BOUND}

\section{Lower bound for the probability}

Let's try to simplify our general equation and put a lower bound to it. Earlier we had ignored all the composite odd numbers, because they did not have any impact on the prime numbers later in the series, and as such have no impact on any potential twin prime number too. Our general equation, as described earlier is

$$
P\left(p_{i}\right)=\frac{\left(p_{i}-2\right)}{p_{i}} * \frac{\left(p_{i-1}-2\right)}{p_{i-1}} * \ldots * \frac{(11-2)}{11} * \frac{(7-2)}{7} * \frac{(5-2)}{5} * \frac{(3-2)}{3}
$$

Let's define a modified probability equation, $M P\left(p_{i}\right)$, which will include all odd numbers (instead of just the prime numbers)

$M P\left(p_{i}\right)=\frac{\left(p_{i}-2\right)}{p_{i}} * \frac{\left(p_{i-1}-2\right)}{p_{i-1}} * \ldots * \frac{(11-2)}{11} * \frac{(9-2)}{9} * \frac{(7-2)}{7} * \frac{(5-2)}{5} * \frac{(3-2)}{3}$

We can assert that $M P\left(p_{i}\right)<=P\left(p_{i}\right)$. The equality holds upto 7 , but once 9 is introduced, $7 / 9<1$, and so on for every composite odd number. Also since this is the minimum probability so far, if we use it across the entire effective range of potential twin primes so far, then we have effectively put a lower bound on the number of expected twin primes. 
Since for the modified probability equation, every $p_{i-1}=\left(p_{i}-2\right)$, this implies that

$$
M P\left(p_{i}\right)=\frac{1}{p_{i}}
$$

\section{Lower bound for the effective range/potential twin primes}

The above probability is valid for all odd numbers $<p_{i+1}^{2}$. Since the minimum difference between two consecutive odd numbers is 2 , we can use $p_{i+1}=p_{i}+2$ to put a lower bound to the effective range.

We had earlier calculated

$$
\operatorname{PTP}(N)=\frac{N+1}{2}-3
$$

not including $\mathrm{N}$

Putting $N=\left(p_{i}+2\right)^{2}$, we get

$$
\begin{gathered}
\operatorname{PTP}\left(\left(p_{i}+2\right)^{2}\right)=\frac{\left(p_{i}+2\right)^{2}+1}{2}-3 \\
\operatorname{PTP}\left(\left(p_{i}+2\right)^{2}\right)=\frac{\left(p_{i}^{2}+4 p_{i}-1\right)}{2}
\end{gathered}
$$

Now we have a lower bound for the probability as well as for the number of potential twin primes overall. The lower bound for the number of twin primes up to the effective range can be determined by multiplying these two.

$$
\begin{gathered}
\text { LowerBound }=M P\left(p_{i}\right) * \operatorname{PTP}\left(\left(p_{i}+2\right)^{2}\right) \\
\text { LowerBound }=\frac{1}{p_{i}} * \frac{\left(p_{i}^{2}+4 p_{i}-1\right)}{2} \\
\text { LowerBound }=\frac{p_{i}}{2}+2-\frac{1}{2 p_{i}}
\end{gathered}
$$

Since we have already dropped the prime number constraint, we can just write the above as

$$
\text { Lower Bound }=\frac{p}{2}+2-\frac{1}{2 p}
$$

where $\mathrm{p}$ is any odd number $>=3$ and the effective range is $<(p+2)^{2}$. 


\section{PROOF}

\section{Using Limits}

Since the equation

$$
\frac{p}{2}+2-\frac{1}{2 p}=\frac{p}{2}+1+\left(1-\frac{1}{2 p}\right)>\frac{p}{2}+1
$$

as $\left(1-\frac{1}{2 p}\right)$ is $>0$.

We can use this and by the definition of limit,

$$
\lim _{p \rightarrow \infty}\left(\frac{p}{2}+1\right)=\infty
$$

Thus there are infinitely many twin primes.

\section{Using Contradiction}

Suppose there are only finitely many twin primes, let's say n of them. We denote them by

$$
t p_{1}, t p_{2}, \ldots, t p_{n}
$$

Now we already know that there are infinitely many prime numbers (or odd numbers). Consider a prime number $\mathrm{p}$ (or an odd number $\mathrm{p}$ ) where $p>2 n$. Using the lower bound for twin primes formula, we get the lower bound of the number of twin primes to be we know that the lower bound of twin primes is given by the formula:

$$
N>=\frac{p}{2}+2-\frac{1}{2 p}
$$

Substituting for $\mathrm{p}$, this becomes

$$
\begin{gathered}
N>\frac{2 n}{2}+2-\frac{1}{4 n} \\
N>\frac{2 n}{2}+1+\left(1-\frac{1}{4 n}\right) \\
N>n+1
\end{gathered}
$$

That's a contradiction, so our original assumption that there are finitely many twin primes must be false. Thus there are infinitely many twin primes. 


\section{CONCLUSION}

We started this work in order to prove the twin prime conjecture. We proved it. And in the process, we ended up also proving that there is at least one twin prime between the squares of not only any two consecutive prime numbers but also between the squares of any two consecutive odd numbers, which can be proved easily since the next odd number will be $p+2$ in the Lower Bound equation. Now that we have established a lower bound for twin primes, we can try to further improve this. We can also use this approach to establish lower bounds for similar conjectures.

\section{REFERENCES}

1. de Polignac, A. (1849). "Recherches nouvelles sur les nombres premiers" [New research on prime numbers]. Comptes rendus (in French). 29: 397-401. From p. 400: "1er Théorème. Tout nombre pair est égal à la différence de deux nombres premiers consécutifs d'une infinité de manières ..." (1st Theorem. Every even number is equal to the difference of two consecutive prime numbers in an infinite number of ways ... )

2. Maynard,James "On The Twin Prime Conjecture," arXiv:1910.14674v1 [math.NT] 29 Oct 2019

3. Yitang Zhang, "Bounded gaps between primes", Ann. of Math., 179,2014 (1121-1174)

Vijay Nandanwar:

Alma mater: Indian Institute of Technology - Kanpur (B.Tech): 1990-94

Indian Institute of Management - Ahmedabad (PGDM): 1995-97

Email address: vijaynandanwar2003@gmail.com 